\title{
The role of habituation and attentional orienting in the disruption of short-term memory performance
}

\author{
Jan Philipp Röer • Raoul Bell • Sandra Dentale • \\ Axel Buchner
}

Published online: 15 January 2011

(C) Psychonomic Society, Inc. 2011

\begin{abstract}
A series of experiments explored habituation and dishabituation to repeated auditory distractors. Participants memorised lists of visually presented items in silence or while ignoring continuously presented auditory distractors. No habituation could be observed, in that the size of the auditory distractor effect did not decrease during the experiment. However, there was evidence for attentional orienting when novel auditory material was presented after a long period of repetitive stimulation, in that a change of distractors was associated with a temporary decrease in recall performance. The results are most consistent with theoretical accounts that claim that the auditory distractor effect is caused primarily by automatic interference, but that still allow attention to play a limited role in the short-term maintenance of information.
\end{abstract}

Keywords Irrelevant speech - Auditory distraction . Immediate memory $\cdot$ Serial recall $\cdot$ Changing-state effect

Serial recall performance for lists of visually or auditorily presented items is impaired in the presence of task-irrelevant background sound (e.g., Beaman, Bridges, \& Scott, 2007; Colle \& Welsh, 1976). This effect is largely independent of the loudness of the distractors (Ellermeier \& Hellbrück, 1998), the phonological and semantic similarity ${ }^{1}$ between the to-be-remembered items and the auditory stimuli (Buchner, Irmen, \& Erdfelder, 1996; Colle \& Welsh, 1976; Salamé \& Baddeley, 1982; but see Bell, Mund, \& Buchner, 2011), and whether the irrelevant material is played accompanying the

J. P. Röer $(\bowtie) \cdot$ R. Bell $\cdot$ S. Dentale $\cdot$ A. Buchner

Department of Experimental Psychology,

Heinrich-Heine-Universität Düsseldorf,

40204 Düsseldorf, Germany

e-mail: jan.roeer@uni-duesseldorf.de target's presentation or subsequently in a retention interval (Buchner, Rothermund, Wentura, \& Mehl, 2004; Miles, Jones, \& Madden, 1991). The irrelevant material's acoustic properties, however, play an important role in determining the size of the auditory distractor effect. The changing-state effect (Jones, Madden, \& Miles, 1992) refers to the phenomenon that a distractor sequence consisting of a single repetitive token generates little or no disruption when compared with a sequence made up from at least two different tokens. Whereas, for example, a single repeated distractor word produces little decrement in performance, an auditory distractor sequence made up of two or more distractor words results in a substantial disruption of serial recall (Bell, Dentale, Buchner, \& Mayr, 2010).

There has been ongoing debate about the involvement of attentional processes in the maintenance of information in working memory. Elliott (2002) suggested that two broad classes of theories of the auditory distractor effect can be distinguished, depending on whether or not they assume that attention plays a role in the short-term maintenance of information (Buchner, Bell, Rothermund, \& Wentura, 2008; Buchner \& Erdfelder, 2005; Buchner, Mehl, Rothermund, \& Wentura, 2006; Buchner et al., 2004; Lange, 2005). Within both the modular working memory model (Baddeley \& Logie, 1999) and the object-oriented episodic record (O-OER) model (Jones, 1993), the auditory distractor effect is explained by assuming that the auditory distractors have automatic access to the representational structure used for the maintenance of the items to be remembered, without a specified role for attention. Within the modular working memory model, the preferred strategy for the immediate serial recall of short visually presented word lists is to convert the words into a phonological representational format for maintenance rehearsal in the limited-capacity phonological loop mod- 
ule. Auditory distractor words automatically gain access to this store and compete with the target representations, thereby impairing recall performance (Salamé \& Baddeley, 1982). Thus, the impairment occurs in a working memory module defined by its phonological representational format, which is why nonphonological properties such as the valence, word frequency, or semantic content of distractors cannot directly affect serial recall. Critically, the component identified with attentional function is the so-called central executive, which is "not involved in temporary storage" (Baddeley \& Logie, 1999, p. 28).

Within the O-OER model, both visually presented to-beremembered items and auditory distractors are represented as amodal objects on a representational structure referred to as the blackboard. Auditory streams are automatically segmented into objects on the basis of abrupt changes in stimulus characteristics. Disruption of serial recall performance occurs because the preattentive seriation of the auditory objects interferes with the intentional maintenance of the to-be-remembered order information. The changingstate effect is explained by assuming that the repeated presentation of the same auditory distractor item results in the representation of a single auditory object on the blackboard, and therefore does not interfere with seriation of the to-be-remembered items. By contrast, the more numerous the occasions on which there is a discrepancy between two successive auditory distractor items, the greater the impairment of serial recall. Thus, within the OOER model, the auditory distractor effect is attributed to automatic interference by seriation processes (e.g., Beaman \& Jones, 1997); a specified role for attention is explicitly excluded. However, a recent modification of the O-OER model, the so-called duplex-mechanism account of auditory distraction (Hughes, Vachon, \& Jones, 2007) proposes that the irrelevant speech effect is mainly caused by automatic and preattentional competition of seriation processes, but also assumes that attention switches to the auditory modality might interfere with the encoding of the target items.

Within the feature model (Nairne, 1990; Neath, 2000), it is assumed that disruption of short-term memory by auditory speech distractors is due to a feature adoption process, according to which features of the auditory distractor sequence overwrite features of the to-beremembered items maintained in working memory. The model, however, also allows for attentional processes playing an important role in the maintenance of working memory information whilst ignoring irrelevant auditory material.

The embedded-processes model (Cowan, 1995, 1999), in contrast, implies a fundamentally different explanation of the auditory distractor effect. According to this framework, working memory comprises a compound of cognitive processes that are necessary to retain information in a highly activated state. Disruption of serial recall performance by distractor speech is explained with attentional recruitment away from the visually presented to-beremembered items, which are rehearsed and kept active in the capacity-limited focus of attention. Novel or changing task-irrelevant stimuli elicit an orienting response, which draws attention away from rehearsal processes and therefore reduces the activation of a target's representation, and thus the probability of successful recall.

Within the embedded-processes framework, habituation of the orienting response (Sokolov, 1963) serves as an attentional filter. The finding that steady-state sequences interfere less with serial recall than do changing-state sequences (Jones et al., 1992) is explained by the fact that the orienting response habituates with repeated stimulus presentation (Thompson \& Spencer, 1966). More precisely, it is assumed that a neural model of the repeated stimulus is formed to which each new incoming stimulus is compared. If discrepancies from the neural model are detected or the neural model is not yet established, an orienting response is elicited. The more times a stimulus sequence is repeated, the more reliably the orienting response should habituate. This is why steady-state distractor sequences (i.e., immediate repetitions of a single distractor item) should be less disruptive than changing-state sequences, but note that the disruption by arbitrary auditory distractors should decrease eventually with repeated presentation.

Recently, empirical evidence has emerged in support of the embedded-processes model and an attentional interpretation of the auditory distractor effect (Bell et al., 2010; Bell et al., 2011; Buchner et al., 2008; Buchner \& Erdfelder, 2005; Buchner et al., 2006; Buchner et al., 2004; Chein \& Fiez, 2010). For instance, Buchner et al. (2006) showed that nonwords that were associated with negative valence in a game context and were later used as auditory distractors caused more disruption of serial recall performance than did neutral nonwords. However, the question of whether attentional factors play a role in the auditory disruption of serial recall is still highly controversial.

Examining whether the auditory distractor effect habituates or not provides a crucial test of the embeddedprocesses model. As mentioned in the previous paragraph, it is known that the orienting reaction-which serves as the explanation for the auditory distractor effect within the embedded-processes model - is subject to habituation (Thompson \& Spencer, 1966). Within the O-OER model, in contrast, it is assumed that the auditory distractor effect is caused by the automatic interference of seriation processes, which are assumed not to habituate. Thus, if the disruption of serial recall were reduced with continuous presentation of a distractor sequence, this would support an attentional interpretation of the auditory distractor effect. If, in 
contrast, the disruption of serial recall remained completely constant in the course of the experiment, this would provide evidence against the embedded-processes model (Cowan, 1995, 1999), and in favour of the O-OER model as suggested by Jones (1993).

However, experiments examining habituation to auditory distractors have yielded inconsistent results. Whereas some studies have shown habituation of the auditory distractor effect (e.g., Banbury \& Berry, 1997; Morris \& Jones, 1990), others have reported no habituation (e.g., Ellermeier \& Zimmer, 1997; Jones, Macken, \& Mosdell, 1997; Tremblay, 1997). On the one hand, it is possible to conclude from these results that the auditory distractor effect is largely independent of attention and that an attentional modulation of the auditory distractor effect occurs only under specific circumstances. On the other hand, it could also be argued that the auditory distractor effect is caused by attentional distraction and actually does habituate, but this habituation is sometimes not detected for methodological reasons.

The latter interpretation is supported by some results that have been interpreted in favour of the O-OER model's predictions and have shown habituation of the auditory distractor effect at a descriptive level. For instance, Jones et al. (1997) examined whether serial recall improves in a changing-state condition with repeated distractor presentation, which would provide evidence for habituation to auditory distractors. Consistent with the predictions of the embedded-processes model, the decrease in recall errors over the course of the experiment was more than three times higher in the changing-state condition than in the quiet control condition. In Jones et al.'s (1997) Experiment 1 , performance improved by 1.23 errors on average from the first to the last trial block in the quiet condition, but by 3.87 errors in the auditory distractor condition. In their Experiment 2, performance improved by 0.67 errors in the quiet condition and by 2.10 errors in the changing-state condition. Nevertheless, in both cases the interaction between distractor condition (quiet vs. auditory distractors) and stimulus repetition (trial block) was not statistically significant. However, with a total sample size of $N=40$ for Experiment 1 and $N=30$ for Experiment 2, the statistical power may have been too small to conclude in favour of the null hypothesis with sufficient confidence.

An additional problem is that evidence against habituation to a repeated stimulus sequence comes from studies using within-subject randomised trial-to-trial manipulations of the distractor condition (e.g., Ellermeier \& Zimmer, 1997). When trials with to-be-habituated distractors alternate with quiet control trials or trials with different types of distractors, this could lead to dishabituation and therefore decrease the probability of finding significant habituation effects.
Other studies have taken an indirect approach to assess habituation of the irrelevant-speech effect by examining effects of the token set size (that is, the number of unique distractor items in a to-be-ignored sequence). From the embedded-processes model, the hypothesis was derived that a distractor sequence consisting of several auditory distractors should disrupt performance more than a sequence consisting of only two alternating distractors. Inconsistent with this assumption, Tremblay and Jones (1998) reported that distractor sequences consisting of two alternating distractors disrupted performance as much as sequences with greater set sizes, which would be inconsistent with the embeddedprocesses model but consistent with the O-OER model. However, sample sizes were comparably small $(n=20$ participants in each group), which makes interpreting nondifferences between conditions problematic, and Campbell and colleagues (Campbell, Beaman, \& Berry, 2002; Campbell, Winkler, Kujala, \& Näätänen, 2003) reported conflicting results. Campbell et al. (2003) found no significant increase of short-term memory disruption when token set size was increased from 1 to 2 , but a significant increase when set size was increased from 2 to 5 . This is inconsistent with the OOER model.

Given these conflicting results, we decided to directly examine habituation to a repeated stimulus sequence in the irrelevant speech paradigm by using measures that provide a fairer test of the habituation hypothesis. First, a betweensubjects manipulation of the distractor condition was used. One group of participants heard series of two alternating one-syllable distractor words (the minimum distractor sound complexity needed to disrupt serial recall reliably), whereas the other group heard no distractors and thus served as the quiet control condition. Second, the statistical power of $1-\beta=.99, .97$, and .93 for Experiments 1, 2, and 3 , respectively, for effects of size $\eta_{p}^{2}=.1$ ensured that possible habituation effects of a reasonable size could be detected with an acceptable probability.

Previous studies had assessed only whether, relative to a quiet control condition, the disruption of short-term memory by auditory distractors decreases with repeated presentations of the same distractor sequence. Such a decrease of disruption need not be due to habituation to the auditory distractors, at least not exclusively. The effect could also be due to the fact that the distractor condition is initially more difficult than the control condition, as a consequence of which the distractor condition has more room for improvement (e.g., by fine-tuning the target rehearsal strategy in the presence of distractors) than the control condition. To ensure that reduced disruption by the distractors is due to habituation, it would be necessary to show that the effect reverses when a new set of auditory distractors is employed. Therefore, the present series of experiments comprised so-called dishabituation trials 
(henceforth, dishabituation refers to a restoration of the disruptive potential of distractors when a habituated speech sequence is replaced by a novel sequence). Specifically, after having completed the habituation trials, participants in both the distractor and the quiet control groups performed a serial recall task while ignoring distractor sequences that changed from trial to trial. An added advantage of this procedure is that it allows us to distinguish the amount of specific habituation that can be traced back to the stimulus identity from a more global form of habituation to more general variables, such as the rhythmic pattern of two onesyllable distractor sounds. If participants in the distractor group habituate to the specific stimulus identity, novel distractor sequences should be more disruptive than if the participants habituate to more general variables.

To sum up, theoretical accounts that claim that the auditory distractor effect is caused by automatic interference (i.e., the modular working memory model, the O-OER model) predict that the auditory distractor effect, relative to a quiet control condition, will remain constant with repeated presentation of the distractor sequence. Furthermore, a change of distractors in the dishabituation trials should have no effect on serial recall performance in the distractor group. By contrast, according to the embedded-processes model, the performance gap between the auditory distractor and the quiet control group should decrease over the course of the experiment, and the change of distractors in the dishabituation trials should result in a performance drop in the distractor group. Theoretical accounts that allow both automatic and attentional processes to play roles in explaining the auditory distractor effect - the feature model and the duplex-mechanism account of auditory distraction (Hughes et al., 2007)_allow for a small modulation of the irrelevant speech effect by attentional disruption that habituates, but they still imply that the interference effect would remain stable over the course of the experiment because the central mechanism that causes the interference does not habituate.

\section{Experiment 1}

In each trial, participants memorised nine single-digit numbers either in silence or while ignoring two alternating auditory distractors. In the auditory distractor condition, the same pair of distractor words was used throughout for 85 trials (and no distractors were played in the quiet condition). Next, all participants recalled series of numbers in five trials, in each of which two new auditory distractors were presented.

Method

Participants A total of 211 students (157 women) at Heinrich-Heine-Universität Düsseldorf were paid for par- ticipating or received course credit. Their ages ranged from 19 to 59 years $(M=26)$. All participants reported normal hearing and normal or corrected-to-normal vision.

Materials For each trial, the order of nine single-digit numbers $(1,2,3,4,5,6,7,8,9)$ was randomised, and the numbers were presented at a rate of 1 per second (on for $800 \mathrm{~ms}$, off for $200 \mathrm{~ms}$ ) in 65-point black Monaco font on a white background in the centre of a computer screen.

The auditory distractor words were 12 German onesyllable nouns (Alm [alp], Elch [moose], Gel [gel], Jod [iodine], Los [lottery ticket], Milz [spleen], Ohm [ohm], Schopf [tuft], Steg [plank], Streu [strewing], Tau [dew], Zwist [strife]). To ensure equal frequencies in the language, these distractor words were selected using the German language corpus available in the CELEX database (Baayen, Piepenbrock, \& van Rijn, 1993). The auditory distractor stimuli were spoken by a female voice and were recorded digitally at $44.1 \mathrm{kHz}$ using 16-bit encoding. The auditory distractors lasted $600 \mathrm{~ms}$ and were normalised to minimise amplitude differences amongst the stimuli. Sounds were produced at a level of about $65 \mathrm{~dB}(\mathrm{~A})$.

Procedure All testing was carried out individually in a sound-attenuating cubicle. The experiment ran on an Apple iMac computer. Participants wore closed headphones throughout the whole experiment. Standard written instructions were given on the computer screen informing the participants to ignore any sound they might hear and to avoid pronouncing the to-be-remembered items.

Each trial began with an introductory period, followed by the to-be-remembered list of nine numbers and a retention interval, during which the computer screen was blank. In the auditory distractor condition, sequences of two alternating distractor words (e.g., “Ohm, Steg, Ohm, Steg, ....”) were presented during the introductory period $(5.4 \mathrm{~s})$, the number presentation ( $9 \mathrm{~s})$, and the retention interval (6s), but not during recall $(9 \mathrm{~s})$. For an illustration of an exemplary auditory distractor trial, see Fig. 1. No auditory distractors were presented in the quiet control condition, but the timing was identical to that of the auditory distractor condition.

A series of question marks, one for each of the serial positions of the items, prompted the forward serial recall. Participants used the keyboard's number pad to enter the numbers in the order in which they had been presented. Each number that was entered replaced one question mark. A position could be omitted by pressing the correspondingly labelled button on the keyboard, as a consequence of which a hyphen replaced the question mark. As in many irrelevant-sound experiments, including our own previous ones (Bell \& Buchner, 2007; Buchner et al., 2008; Buchner \& Erdfelder, 2005; Buchner et al., 1996; Buchner et al., 2004), participants were allowed to correct their responses 


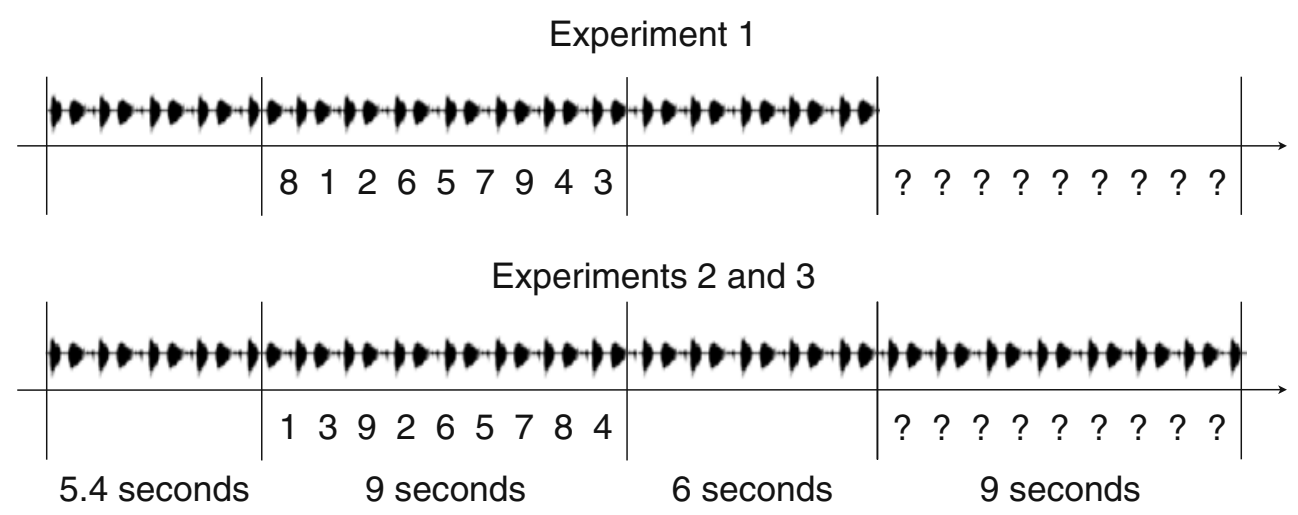

Fig. 1 Illustration of a trial with auditory distractors. In Experiment 1, auditory distractors were only presented during the introductory period (5.4 s), the presentation of to-be-remembered items $(9 \mathrm{~s})$, and the retention interval ( $6 \mathrm{~s})$, but not during recall (9 s). In Experiments 2 and 3, auditory distractors were presented continuously without periods of silence using the arrow keys to move to an arbitrary position and to replace any prior entry. Note that participants were nevertheless required to recall items in forward order, because they had to replace the first question mark with a digit or a "don't know" response before they were allowed to continue with replacing the second question mark, and so on. After $6 \mathrm{~s}$, a visually presented countdown indicated the number of seconds $(3,2,1)$ left for recall, after which the next trial was initiated automatically.

For the auditory distractor group, a sequence of two alternating distractor words (e.g., "Ohm, Steg, Ohm, Steg, ..."; henceforth $A B$ ) was played during Trials 1-85, except for the recall interval. Participants in the quiet condition completed the first 85 trials in silence. Trials 86-90 were parallel for the auditory distractor and the control groups. In each of these trials, a different pair of words formed the sequence of alternating distractor words (e.g., "Alm, Jod, Alm, Jod, ..." in Trial 86, referred to as CD in Fig. 3; "Tau, Los, Tau, Los, . . ." in Trial 87, referred to as EF in Fig. 3; etc.). For each participant, all auditory distractor words were selected randomly without replacement from the set of possible distractor words introduced in the Materials section.

The experiment took approximately $50 \mathrm{~min}$ to complete, after which participants were offered an explanation as to the purpose of the experiment.

Design For the traditional approach to analyse habituation, the first 80 trials were split into four blocks consisting of 20 trials each. Thus, a mixed design with block $(1,2,3,4)$ as a within-subjects variable and auditory condition (auditory distractor, quiet) as a between-subjects variable was used. A progressively smaller gap in recall performance between the auditory distractor and quiet control groups would be important evidence in favour of the assumption that participants habituated to the distractor word sequences.
Given a total sample size of $N=211$ (with random group assignments leading to $n=104$ in the auditory distractor group and $n=107$ in the quiet control group) and $\alpha=.05$, a block $(1,2,3,4) \mathrm{x}$ auditory condition (auditory distractor, quiet) interaction of size $\eta_{p}^{2}=.1$ could be detected with a probability of $1-\beta=.99$.

The level of $\alpha$ was set to .05 for all analyses. Partial $\eta^{2}$ is reported as a measure of effect size. All power calculations reported in this article were conducted using $\mathrm{G}^{*}$ Power (Faul, Erdfelder, Lang, \& Buchner, 2007).

\section{Results}

Recall was scored as correct when numbers were reproduced in the correct position. Participants had $9 \mathrm{~s}$ to enter the recalled numbers before the next trial began. Only $1.4 \%$ of the question marks across all three experiments were not replaced by numbers or by the hyphen that was used to indicate that a number could not be recalled. This shows that the 9-s recall interval was sufficiently long in all experiments reported in this article.

Figure 2 (upper panel) illustrates the serial recall performance across the four blocks of habituation trials in the auditory distractor and the quiet control groups. There was a main effect of auditory condition, $F(1,209)=24.50$, $p<.001, \eta_{p}^{2}=.11$, confirming that there was an irrelevantspeech effect. Replicating previous studies (Ellermeier \& Zimmer, 1997; Jones, Macken, \& Mosdell, 1997; Tremblay \& Jones, 1998), participants' performance improved from Blocks 1 to $4, F(3,207)=76.23, p<.001, \eta_{p}^{2}=.53$, suggesting that they benefitted from familiarization with the task. However, there was no significant interaction between the auditory condition and block variables, $F(3,207)=$ $0.70, p=.552, \eta_{p}^{2}=.01$, and thus no evidence for habituation of the auditory distractor effect. 

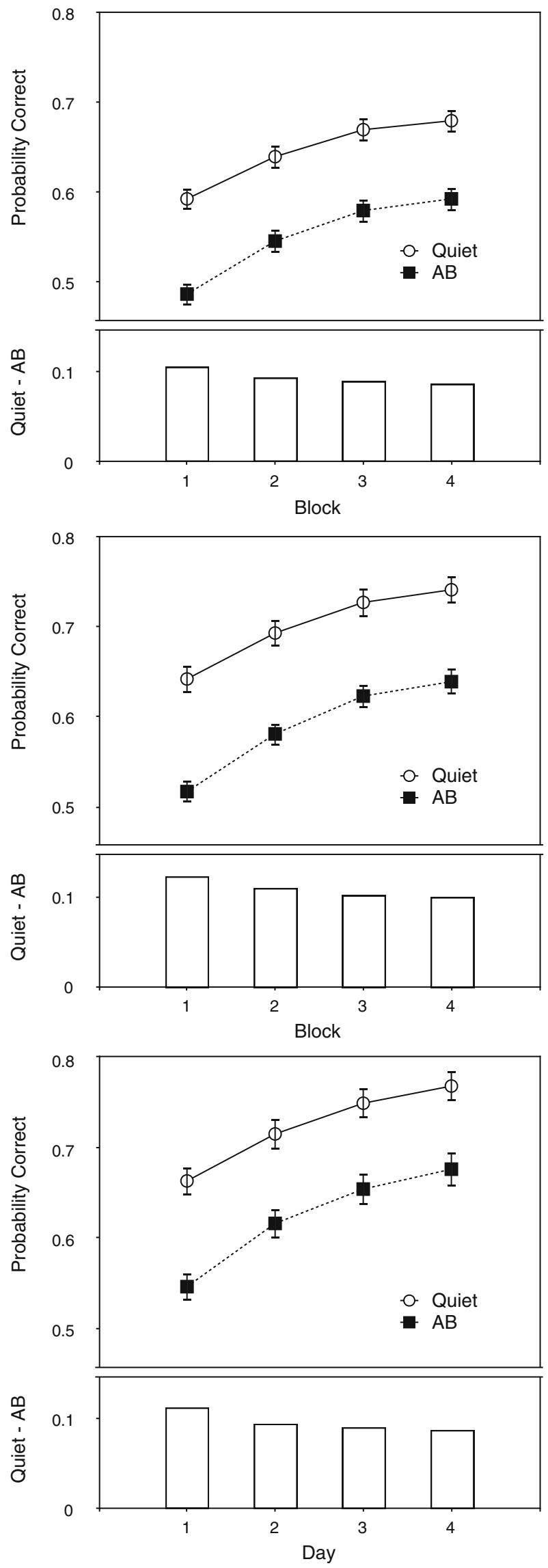

Fig. 2 Serial recall performance across four blocks of 20 trials each (spanning Trials 1-80) for the auditory distractor group (AB) and the quiet control group (Quiet) in Experiments 1 (upper panel), 2 (middle panel), and 3 (lower panel). The error bars depict the standard errors of the means. The column graphs below the line graphs represent the performance differences between the auditory distractor group and the quiet control group

The left and right halves of Fig. 3 (upper panel) illustrate the serial recall performance for Trials $81-85$ (with no distractors for the control group and the same distractors as in the preceding trials for the auditory distractor group) and for Trials 86-90 (with a new pair of distractors in every trial for both groups), respectively. Of interest is whether the change in the distractor sequence led to a drop in performance. Indeed, participants in the auditory distractor condition made more serial recall errors in Trial 86, as compared to the final five habituation trials (Trials 81-85) combined, $F(1,103)=4.84, p=.030, \eta_{p}^{2}=.05$. This could be interpreted by assuming that the change of the distractor sequence led to an orienting reaction, which recruited attentional processes away from serial recall. Interestingly, serial recall performance recovered in the trials in which randomly selected new pairs of distractors were used; the contrast between Trial 86 and Trials 87-90 combined was significant, $F(1,103)=5.26, p=.024, \eta_{p}^{2}=.05$. The same applies to the quiet control group, in which the contrast between Trial 86 and Trials $87-90$ combined was also significant, $F(1,106)=17.89, p<.001, \eta_{p}^{2}=.14$.

\section{Discussion}

Participants in the quiet control condition recalled more items correctly than did participants in the auditory distractor condition, reflecting the fact that the auditory distractor effect is a robust and stable phenomenon. There was no statistically significant decrease in this performance gap between the auditory distractor and quiet control groups, which is consistent with previous experiments examining habituation to a repeated stimulus sequence (Ellermeier \& Zimmer, 1997; Jones et al., 1997). However, the straightforward interpretation of this data pattern in favour of the assumption that the auditory distractor effect shows no sign of habituation is complicated by two aspects of the present data. First, at least descriptively, the difference between the quiet control and auditory distractor groups became progressively smaller (Fig. 2, upper panel, column bars). Second, serial recall performance of participants in the auditory distractor group dropped significantly when the pair of distractor words changed in Trial 86 as compared to performance in the preceding five trials. (The fact that performance recovered in subsequent trials, although the composition of the distractor sequences changed from trial to trial, will be considered in the General Discussion section.) These two aspects of the 

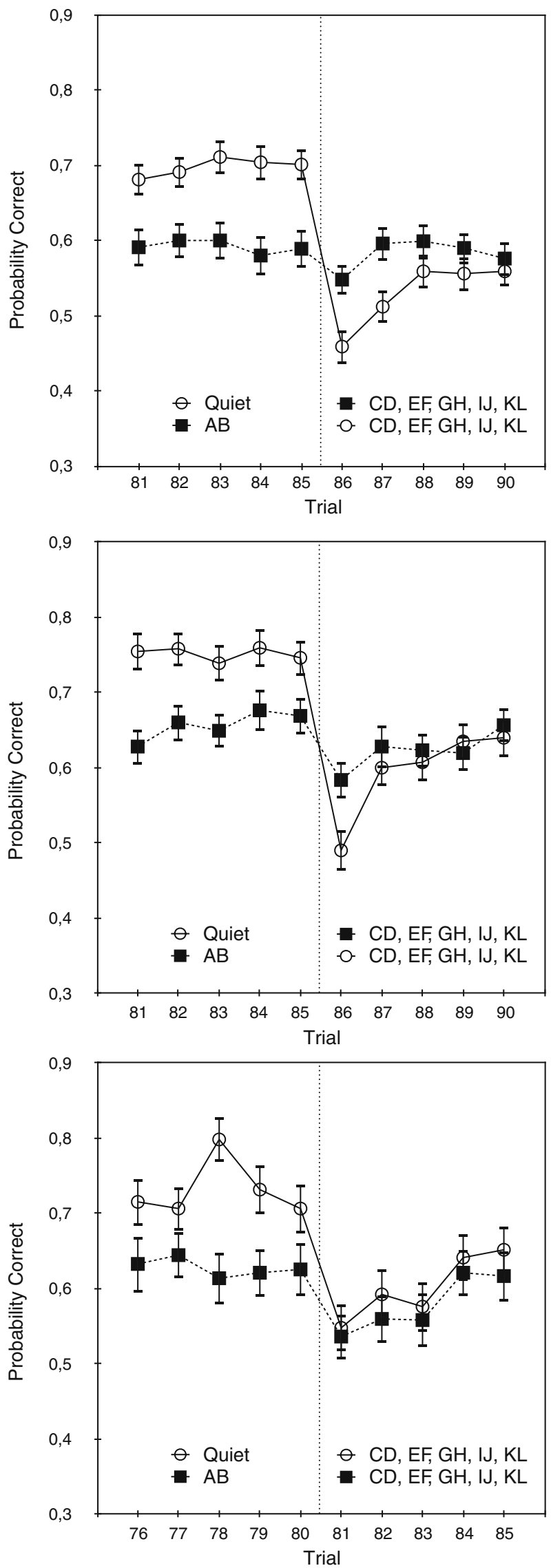

Fig. 3 Serial recall performance in the final five habituation trials (Trials 81-85) and the five dishabituation trials (Trials 86-90) for the auditory distractor group (AB for Trials $81-85$; CD, EF, GH, IJ, KL for Trials 86-90) and the quiet control group (Quiet for Trials 81-85; CD, EF, GH, IJ, KL for Trials 86-90) in Experiments 1 (upper panel), 2 (middle panel), and 3 (lower panel). The error bars depict the standard errors of the means

present data suggest that habituation may have occurred, but that it may not have been sufficiently strong to be reflected in the serial recall measure during the habituation trials. Consider, for instance, that participants in the auditory distractor group experienced periods of silence when they recalled the sequence of numbers. It seems possible that these periods of silence caused dishabituation. This idea gains plausibility from the finding of Banbury and Berry (1997) that a period of silence can cause an effect of dishabituation in participants previously habituated to irrelevant speech. To eliminate this possible source of dishabituation, the auditory distractors were played continuously and without any interruption in Experiment 2. This should not affect the size of the auditory distractor effect (see, e.g., Miles et al., 1991), but it might lead to a reduction of the performance gap between the quiet control and auditory distractor groups as a function of the number of habituation trials.

\section{Experiment 2}

\section{Method}

Participants A total of 182 students (138 women) at Heinrich-Heine-Universität Düsseldorf were paid for participating or received course credit. Their ages ranged from 18 to 50 years $(M=26)$. All participants reported normal hearing and normal or corrected-to-normal vision.

Materials, procedure, and design Experiment 2 was identical to Experiment 1, except that in trials with auditory distractors, the alternating word sequences continued to be played during recall, so that there were no silent periods between trials. For an illustration of an auditory distractor trial, see Fig. 1. Given a total sample size of $N=182$ (with random group assignments leading to $n=90$ in the auditory distractors group and $n=92$ in the quiet control group) and $\alpha=.05$, a reduction of the performance gap between the quiet control and the auditory distractor group of size $\eta_{p}^{2}=$ .1 could be detected with a probability of $1-\beta=.97$.

\section{Results}

Figure 2 (middle panel) shows that, as in Experiment 1, serial recall performance increased from Blocks 1 to $4, F(3$, 
178) $=79.38, p<.001, \eta_{p}^{2}=.57$, and was better in the quiet control then in the auditory distractor condition, $F(1$, $180)=34.12, p<.001, \eta_{p}^{2}=.16$. However, there was again no interaction between the auditory condition and block variables, $F(3,178)=0.92, p=.431, \eta_{p}^{2}=.02$, and thus, no evidence for habituation.

The left half of Fig. 3 (middle panel) shows that, as in Experiment 1, recall performance in the auditory distractor group was significantly reduced in Trial 86 (the first trial with a new pair of distractors) compared to Trials $81-85$ combined, $F(1,89)=10.48, p=.002, \eta_{p}^{2}=.11$. Performance again recovered in the subsequent trials, in which randomly selected new pairs of distractors were used. The contrast between Trial 86 and Trials 87-90 combined was significant, $F(1,89)=4.39, p=.039, \eta_{p}^{2}=.05$. The same applies to the quiet control group, in which the contrast between Trial 86 and Trials $87-90$ combined was also significant, $F(1,91)=33.84, p=<.001, \eta_{p}^{2}=.27$. Thus, the results are parallel to those of Experiment 1.

\section{Discussion}

The results of Experiment 2 conceptually replicated those of Experiment 1 in showing that continuous exposure to auditory distractor sequences did not result in a statistically significant reduction of the performance gap between the auditory distractor and quiet control groups. Thus, there seems to be no evidence for habituation of the auditory distractor effect in this data pattern. However, as in Experiment 1, the difference between the quiet control and auditory distractor groups tended to become progressively smaller at a descriptive level (Fig. 2, middle panel, column bars). Second, serial recall performance in the auditory distractor group dropped significantly when the pair of distractor words changed in Trial 86 (the first trial with a different pair of distractors). Thus, there are again hints that a certain amount of habituation may have been occurring as a function of prolonged exposure to the auditory distractors.

In Experiment 3, we went one step further and tested the assumption that it may not be possible to develop a sufficiently complete and stable neural model that represents all stimulus characteristics accurately in a single session (Cowan, 1995). Therefore, in Experiment 3, the four experimental blocks were completed on different days to allow for consolidation to take place. If habituation had been too small to affect recall performance in Experiments 1 and 2 because substantial habituation requires a much more solid representation of the to-be-habituated distractor in long-term memory, the performance gap between the quiet control group and the auditory distractor group should be more pronounced in Experiment 3 than in Experiment 2.

\section{Experiment 3}

Method

Participants A total of 151 undergraduate students (117 women) at Heinrich-Heine-Universität Düsseldorf were paid for participating or received course credit. Their ages ranged from 18 to 50 years $(M=25)$. All participants reported normal hearing and normal or corrected-to-normal vision. Five participants were dropped from the analysis for failure to complete the 4 days.

Materials, procedure, and design Experiment 3 was identical to Experiment 2, except that participants completed four habituation blocks of 20 trials each on four consecutive weekdays (Monday to Thursday or Tuesday to Friday). Five more trials using the same continuously played distractor pair (Trials 81-85) were completed on Day 4, followed by five trials in each of which a randomly selected new pair of distractors was used (Trials 86-90). Given a total sample size of $N=146$ (with random group assignments leading to $n=73$ in the auditory distractor group and $n=73$ in the quiet control group) and $\alpha=.05$, a reduction of the performance gap between the quiet control and auditory distractor groups of size $\eta_{p}^{2}=.1$ could be detected with a probability of $1-$ $\beta=.93$.

\section{Results}

Figure 2 (lower panel) shows that, as in Experiments 1 and 2 , serial recall performance increased from the 1 st to the 4 th block (or weekday), $F(3,142)=84.03, p<.001, \eta_{p}^{2}=.64$, and was better in the quiet control then in the auditory distractor condition, $F(1,144)=19.63, p<.001, \eta_{p}^{2}=.12$ There was again no interaction between auditory condition and block (or weekday), $F(3,142)=1.10, p=.352$, $\eta_{p}^{2}=.02$.

The left half of Fig. 3 (lower panel) shows that, as in Experiments 1 and 2, recall performance in the auditory distractors group was significantly reduced in Trial 86 (the first trial with a new pair of distractors) compared to Trials 81-85 combined, $F(1,72)=13.31 p<.001$, $\eta_{p}^{2}=.16$. As in the previous experiments, the performance of participants in the auditory distractors group recovered in the trials in which randomly selected new pairs of distractors were used. The contrast between the Trial 86 and Trials 87-90 combined was again significant, $F(1,72)=5.45, p=.022, \eta_{p}^{2}=.07$. The same applies to the quiet control group, in which the contrast between Trial 86 and Trials 87-90 combined was also significant, $F(1,72)=6.16, p=.015, \eta_{p}^{2}=.08$. 


\section{Discussion}

Even with the possibility of consolidation occurring between blocks of trials, the results of Experiment 3 perfectly replicated the findings of Experiments 1 and 2 . The continuous exposure to the same auditory distractors on consecutive weekdays did not result in a statistically significant reduction of the performance gap between the auditory distractor and quiet control groups. Again, however, this gap decreased descriptively (Fig. 2, lower panel, column bars), and there was again a significant drop in performance when the repeated distractor sequence suddenly changed in Trial 86. Consistent with Experiments 1 and 2, participants in the auditory distractor condition made more errors in recall when a new set of stimuli was used as in the preceding habituation trials. Thus, as in Experiments 1 and 2, there is some evidence for habituation to auditory distractors, but only in a subset of the measures that were available in the experiments reported here.

\section{General discussion}

The results obtained in the present series of experiments are very consistent and were unaffected by whether there were periods of silence during recall or whether there was an opportunity for consolidation. First and foremost, the performance gap in serial recall between a group who received the same pair of alternating one-syllable spoken distractor words and a quiet control group was not significantly reduced even within four blocks of 80 trials each (or 1,960 repetitions of the distractor word pair in Experiments 2 and 3). Given that other studies found fast habituation of the orienting response over the first few trials (Elliott \& Cowan, 2001; Shelton, Elliott, Eaves, \& Exner, 2009), we performed a supplementary analysis to examine habituation across the trials in the first block. This analysis showed that there was also no evidence of a fast initial (Trials 1-5) reduction of the irrelevant-speech effect in Experiments $1[F(4,206)=0.59, p=.669$, $\left.\eta_{p}^{2}=.01\right], 2\left[F(4,177)=2.35, p=.056, \eta_{p}^{2}=.05\right]$, and 3 $\left[F(4,141)=0.99, p=.413, \eta_{p}^{2}=.03\right]$. There was also no evidence of a reduction when we compared the interference effect in the first and second halves of the first block in Experiments $1\left[F(1,209)=0.01, p=.917, \eta_{p}^{2} .01\right], 2[F(1$, $\left.180)=0.01, p=.914, \eta_{p}^{2} .01\right]$, and $3[F(1,144)=0.34, p=$ $.564, \eta_{p}^{2} .01$ ], respectively. Second, at a descriptive level there was a small but nonsignificant reduction of the irrelevantspeech effect across blocks in all experiments. Third, when the distractors changed, performance dropped quite substantially.

The most important finding of the present experiments is the absence of habituation in the irrelevant-speech paradigm. Habituation was absent even though large sample sizes (544 participants in total) guaranteed high statistical power and even though a between-subjects design was used to prevent dishabituation. This finding was replicated even when the distractor sequences were played continuously (in Experiments 2 and 3 ) and when the procedure allowed for consolidation of the distractor information (Experiment 3). This finding is consistent with previous studies reporting no habituation to repeated stimulus sequences (Ellermeier \& Zimmer, 1997; Jones et al., 1997). The absence of habituation is especially interesting given that habituation to auditory distractors has been observed in cross-modal attention paradigms employing primary tasks other than immediate serial recall (Banbury \& Berry, 1997; Elliott \& Cowan, 2001; Shelton et al., 2009), which suggests that the disruption of immediate serial recall by irrelevant speech is a special type of interference effect with a comparably small component of attentional distraction.

It is this aspect of the present data that seems problematic for the embedded-processes model (Cowan, 1995, 1999). If the explanation of impaired serial recall performance in the presence of auditory distractors is assumed to depend entirely on the eliciting of orienting responses by novel or changing task-irrelevant stimuli that draw attention away from rehearsal processes, the amount of disruption should be reduced after prolonged exposure to the same spoken distractor word pair due to habituation. To be sure, there was a descriptive trend towards a reduced performance gap between the auditory distractor group and the quiet control group with repeated exposure to the auditory distractors. However, the trend was rather small and was far from being statistically significant, despite the fact that the statistical power to detect possible habituation processes was substantial in the present experiments.

The finding of no habituation of the irrelevant-speech effect is consistent with models that assume that the effect is caused by automatic interference, such as the modular working memory model and the O-OER model. For instance, the O-OER model proposes that the visually presented to-be-remembered numbers and spoken words are represented as separate streams of amodal objects in one representational structure. Disruption of serial recall performance is assumed to occur because the preattentive seriation of the auditory distractors interferes with the seriation process needed to maintain the to-be-remembered information of the order of the numbers. The model provides no mechanism by which this seriation conflict could be reduced as a function of exposure to the same distractors, and thus it should stay constant even after 80 trials of exposure to the same pair of distractor words.

Nevertheless, performance dropped considerably when distractor identity changed in the dishabituation trials. This finding is consistent with earlier findings that attention plays a role in the maintenance of information in working memory (Buchner et al., 2008; Buchner \& Erdfelder, 2005; 
Buchner et al., 2004; Neely \& LeCompte, 1999). The embedded-processes model (Cowan, 1995, 1999) would explain this finding with an attentional orienting response that is elicited when a discrepancy to a previously formed neural model is detected. Similarly, the feature model can account for this data pattern (Nairne, 1990; Neath, 2000) simply by assuming that novel distractors attract processing resources away from the primary memorisation task. In contrast, the modular working memory model cannot explain the present findings, because it assumes that the component identified with attentional function is the socalled central executive, which is "not involved in temporary storage" (Baddeley \& Logie, 1999, p. 28). We have already explicated elsewhere why we are sceptical that this model can be extended to explain findings such as those presented here and elsewhere (Buchner et al., 2008), which is why we will not repeat these arguments here.

The original O-OER model (Jones, 1993) also cannot explain the sudden drop in performance when the distractors changed. However, this model has recently been extended successfully to include a mechanism by which working memory would remain open to interruption by previously unattended but potentially important information (Hughes, Vachon, \& Jones, 2005; Hughes et al., 2007). Serial recall would then suffer not only from competing seriation processes within the representational structure used to temporarily maintain information (causing the reduction of serial recall performance by auditory distractors), but also from attention distraction to task-irrelevant events (causing temporary distraction, and thus temporary reductions of serial recall performance). This duplex account has recently received some independent support (Klatte, Lachmann, Schlittmeier, \& Hellbrück, 2010; Sorqvist, 2010). It is obviously more complex, and thus somewhat less elegant, than the original O-OER model, but it has the advantage of providing a straightforward explanation for the attentional capture when distractor identity changed and for the absence of habituation of the irrelevant-speech effect across a large number of trials.

Interestingly, just like the recent modifications of the OOER model (Hughes et al., 2005, 2007), the feature model (Nairne, 1990; Neath, 2000) also relies on a duplex mechanism to explain performance decrements in immediate serial recall. As mentioned above, the model contains the assumption that processing resources are necessary for rehearsal in short-term memory, and it may be assumed that novel distractors attract processing resources away from the primary memorisation task. However, the "typical" disruption of serial recall by auditory distractors is caused by an automatic process in which features of to-be-ignored distractors may overwrite features of the to-beremembered targets, which degrades the target representations in working memory so that their probability of being available for correct serial recall is reduced. There is no reason to assume that this automatic process of feature overwriting should habituate, which is why the performance gap between the auditory distractor and quiet control groups should stay constant, even for 80 trials with the same two alternating auditory distractors.

Returning to the data observed in the present series of experiments, perhaps the most surprising aspect was that after an initial and considerable drop in performance when the pair of distractor words changed in Trial 86, recovery was basically complete after two or three more trials. This complete recovery is remarkable because the pair of distractor words was no longer constant but changed from trial to trial in Trials 86-90. Thus, the process that attracts processing resources away from the primary task (feature model; cf. Nairne, 1990; Neath, 2000) or causes attentional capture (modified O-OER model; cf. Hughes et al., 2005, 2007) is obviously much more powerful and flexible than one would assume at first. In the present experiments, the process seems to have adapted first to constant and predictable distractor identities (two alternating spoken words such as "Ohm, Steg, Ohm, Steg, ...") during Trials $1-85$, as is evidenced by the disruption in performance when these identities changed in Trial 86. It seems to have adapted again subsequently, but due to the fact that the distractor identities changed from trial to trial, it must have adapted to general distractor features, such as the constant rhythmic pattern implemented by the alternating onesyllable words or the speaker's voice (or both). In essence, then, habituation of the auditory distractor effect is both much smaller than one would expect from the point of view of the embedded-processes model, in that the performance gap between the quiet control and auditory distractor conditions is hardly (if at all) reduced, and much more powerful, in that attentional capture may be eliminated quickly even if only relatively general distractor features such as rhythmicity or voice characteristics stay constant. The fast adaptability of the attentional system is also evident from other studies examining habituation in crossmodal paradigms in which rapid recoveries from the attentional orienting response have been observed (Elliott \& Cowan, 2001; Shelton et al., 2009).

In summary, the present series of experiments shows that that the irrelevant-speech effect does not habituate. Nevertheless, there was a decrement in performance when distractor identity changed after repeated exposure to the same distractor pair. Together, the results provide evidence both against working memory models that do not allow attention to play a role in the short-term maintenance of information, and against explanations that attribute the irrelevant speech effect exclusively to attentional distraction. Thus, the present results can only be explained by models that assume that the irrelevant-speech effect is 
caused primarily by automatic interference but that still allow attention to play a role in the disruption of short-term memory - that is, by models such as the feature model (Nairne, 1990; Neath, 2000) and the duplex-mechanism account proposed by Hughes et al. $(2005,2007)$.

\section{References}

Baayen, R. H., Piepenbrock, R., \& van Rijn, H. (1993). The CELEX lexical database (Release 1) [CD-ROM]. Philadelphia: Linguistic Data Consortium, University of Pennsylvania [Distributor].

Baddeley, A., \& Logie, R. (1999). The multiple-component model. In A. Miyake \& P. Shah (Eds.), Models of working memory. Cambridge: Cambridge University Press.

Banbury, S., \& Berry, D. C. (1997). Habituation and dishabituation to speech and office noise. Journal of Experimental Psychology. Applied, 3, 181-195.

Beaman, C. P. (2004). The irrelevant sound phenomenon revisited: What role for working memory capacity? Journal of Experimental Psychology. Learning, Memory, and Cognition, 30, 1106-1118.

Beaman, C. P., Bridges, A. M., \& Scott, S. K. (2007). From dichotic listening to the irrelevant sound effect: A behavioural and neuroimaging analysis of the processing of unattended speech. Cortex, 43, 124-134.

Beaman, C. P., \& Jones, D. M. (1997). Role of serial order in the irrelevant speech effect: Tests of the changing-state hypothesis. Journal of Experimental Psychology. Learning, Memory, and Cognition, 23, 459-471.

Bell, R., \& Buchner, A. (2007). Equivalent irrelevant-sound effects for old and young adults. Memory \& Cognition, 35, 352-364.

Bell, R., Buchner, A., \& Mund, I. (2008). Age-related differences in irrelevant-speech effects. Psychology and Aging, 23, 377-391.

Bell, R., Dentale, S., Buchner, A., \& Mayr, S. (2010). ERP correlates of the irrelevant sound effect. Psychophysiology, 47, 1182-1191.

Bell, R., Mund, I., \& Buchner, A. (2011). Disruption of short-term memory by distractor speech: Does content matter? Quarterly Journal of Experimental Psychology, 64, 146-168.

Buchner, A., Bell, R., Rothermund, K., \& Wentura, D. (2008). Sound source location modulates the irrelevant sound effect. Memory \& Cognition, 36, 617-628.

Buchner, A., \& Erdfelder, E. (2005). Word frequency of irrelevant speech distractors affects serial recall. Memory \& Cognition, 33, 86-97.

Buchner, A., Irmen, L., \& Erdfelder, E. (1996). On the irrelevance of semantic information for the "irrelevant speech" effect. The Quarterly Journal of Experimental Psychology, 49A, 765-779.

Buchner, A., Mehl, B., Rothermund, K., \& Wentura, D. (2006). Artificially induced valence of distractor words increases the effects of irrelevant speech on serial recall. Memory \& Cognition, 34, 1055-1062.

Buchner, A., Rothermund, K., Wentura, D., \& Mehl, B. (2004). Valence of distractor words increases the effects of irrelevant speech on serial recall. Memory \& Cognition, 32, 722-731.

Campbell, T., Beaman, C. P., \& Berry, D. C. (2002). Changing-state disruption of lip-reading by irrelevant sound in perceptual and memory tasks. European Journal of Cognitive Psychology, 14, 461-474.

Campbell, T., Winkler, I., Kujala, T., \& Näätänen, R. (2003). The N1 hypothesis and irrelevant sound: Evidence from token set size effects. Cognitive Brain Research, 18, 39-47.

Chein, J. M., \& Fiez, J. A. (2010). Evaluating models of working memory through the effects of concurrent irrelevant information. Journal of Experimental Psychology: General, 139, 117-137.
Colle, H. A., \& Welsh, A. (1976). Acoustic masking in primary memory. Journal of Verbal Learning and Verbal Behavior, 15, $17-31$.

Cowan, N. (1995). Attention and memory: An integrated framework. New York: Oxford University Press.

Cowan, N. (1999). An embedded-processes model of working memory. In A. Miyake \& P. Shah (Eds.), Models of working memory: Mechanisms of active maintenance and executive control (pp. 62-101). New York: Cambridge University Press.

Ellermeier, W., \& Hellbrück, J. (1998). Is level irrelevant in "irrelevant speech"? Effects of loudness, signal-to-noise ratio, and binaural unmasking. Journal of Experimental Psychology: Human Perception and Performance, 24, 1406-1414.

Ellermeier, W., \& Zimmer, K. (1997). Individual differences in susceptibility to the "irrelevant speech effect". The Journal of the Acoustical Society of America, 102, 2191-2199.

Elliott, E. M. (2002). The irrelevant-speech effect and children: Theoretical implications of developmental change. Memory \& Cognition, 30, 478-487.

Elliott, E. M., \& Cowan, N. (2001). Habituation to auditory distractors in a cross-modal, color-word interference task. Journal of Experimental Psychology. Learning, Memory, and Cognition, 27, 654-667.

Faul, F., Erdfelder, E., Lang, A.-G., \& Buchner, A. (2007). G*Power 3: A flexible statistical power analysis program for the social, behavioral, and biomedical sciences. Behavior Research Methods, 39, 175-191.

Hughes, R. W., Vachon, F., \& Jones, D. M. (2005). Auditory attentional capture during serial recall: Violations at encoding of an algorithm-based neural model? Journal of Experimental Psychology. Learning, Memory, and Cognition, 31, 736-749.

Hughes, R. W., Vachon, F., \& Jones, D. M. (2007). Disruption of shortterm memory by changing and deviant sounds: Support for a duplexmechanism account of auditory distraction. Journal of Experimental Psychology. Learning, Memory, and Cognition, 33, 1050-1061.

Jones, D. M. (1993). Objects, streams, and threads of auditory attention. In L. Weiskrantz \& A. D. Baddeley (Eds.), Attention: Selection, awareness, and control. A tribute to Donald Broadbent (pp. 87-104). Oxford University Press: New York.

Jones, D. M., Macken, W. J., \& Mosdell, N. A. (1997). The role of habituation in the disruption of recall performance by irrelevant sound. British Journal of Psychology, 88, 549-564.

Jones, D. M., Madden, C., \& Miles, C. (1992). Privileged access by irrelevant speech to short-term memory: The role of changing state. The Quarterly Journal of Experimental Psychology, 44A, 645-669.

Klatte, M., Lachmann, T., Schlittmeier, S., \& Hellbrück, J. (2010). The irrelevant sound effect in short-term memory: Is there developmental change? European Journal of Cognitive Psychology, 22, 1168-1191.

Lange, E. (2005). Disruption of attention by irrelevant stimuli in serial recall. Journal of Memory and Language, 53, 513-531.

Marsh, J. E., Hughes, R. W., \& Jones, D. M. (2008). Auditory distraction in semantic memory: A process-based approach. Journal of Memory and Language, 58, 682-700.

Marsh, J. E., Vachon, F., \& Jones, D. M. (2008). When does betweensequence phonological similarity promote irrelevant sound disruption? Journal of Experimental Psychology. Learning, Memory, and Cognition, 34, 243-248.

Miles, C., Jones, D. M., \& Madden, C. A. (1991). Locus of the irrelevant speech effect in short-term memory. Journal of Experimental Psychology. Learning, Memory, and Cognition, 17, 578-584.

Morris, N., \& Jones, D. M. (1990). Habituation to irrelevant speech: Effects on a visual short-term memory task. Perception \& Psychophysics, 47, 291-297.

Nairne, J. S. (1990). A feature model of immediate memory. Memory \& Cognition, 18, 251-269. 
Neath, I. (2000). Modeling the effects of irrelevant speech on memory. Psychonomic Bulletin \& Review, 7, 403-423.

Neely, C. B., \& LeCompte, D. C. (1999). The importance of semantic similarity to the irrelevant speech effect. Memory \& Cognition, 27, 37-44.

Salamé, P., \& Baddeley, A. D. (1982). Disruption of short-term memory by unattended speech: Implications for the structure of working memory. Journal of Verbal Learning and Verbal Behavior, 21, 150-164.

Shelton, J. T., Elliott, E. M., Eaves, S. D., \& Exner, A. L. (2009). The distracting effects of a ringing cell phone: An investigation of the laboratory and the classroom setting. Journal of Environmental Psychology, 29, 513-521.

Sokolov, E. N. (1963). Perception and the conditioned reflex. New York: Pergamon Press.

Sorqvist, P. (2010). High working memory capacity attenuates the deviation effect but not the changing-state effect: Further support for the duplex-mechanism account of auditory distraction. Memory \& Cognition, 38, 651-658.
Thompson, R. F., \& Spencer, W. A. (1966). Habituation: A model phenomenon for the study of neuronal substrates of behavior. Psychological Review, 73, 16-43.

Tremblay, S. (1997). Is there a relationship between habituation to irrelevant sound effect and the spectral complexity of auditory items? In A. Schick \& M. Klatte (Eds.), Contributions to psychological acoustics (pp. 521-530). Oldenburg, Germany: BIS.

Tremblay, S., \& Jones, D. M. (1998). Role of habituation in the irrelevant sound effect: Evidence from the effects of token set size and rate of transition. Journal of Experimental Psychology. Learning, Memory, and Cognition, 24, 659-671.

This research was supported by Grant BU 945/8-1 from the German Research Foundation (DFG). 